\title{
El valor del aprendizaje incidental en la toma de decisión y control motor en baloncesto
}

\section{Incidental learning value in basketball decision making and control motor}

\author{
Pablo Camacho
}

\begin{abstract}
PALABRAS CLAVE: Aprendizaje incidental, toma de decisión, control motor, rendimiento, baloncesto.

RESUMEN: En la mayor parte de las situaciones que se producen en el baloncesto, el jugador debe tomar decisiones en un momento concreto de una fracción de tiempo muy limitada, mientras de forma simultánea ejecuta respuestas motoras también inmediatas. Son reacciones motoras rápidas tomadas en situación de presión, donde no tiene tiempo para reflexionar (Kibele, 2006). Estas decisiones parecen basarse en la intuición (Iglesias, Cárdenas \& Alarcón, 2007) y se relacionan con el aprendizaje incidental (Poolton, Masters \& Maxwell, 2006). En un deporte como el baloncesto donde el tiempo es muy reducido y se necesita ejecutar de manera simultánea una respuesta motora inmediata, los procesos intuitivos parecen que pueden proporcionar la exactitud y la rapidez deseada (Raab \& Johnson, 2008), favoreciendo que se produzca una toma de decisión eficaz en condición de estrés, con límite de tiempo y poca información. Teniendo en cuenta que los procesos deliberados y automáticos funcionan simultáneamente y que en su conjunto forman el pensamiento y la acción (Betsch, 2008), nosotros abogamos por un modelo integrado que tenga en cuenta a ambos, pues como afirman Sun, Slusarz \& Terry (2005), los procesos incidentales e intencionales coexisten e interactúan para formar el aprendizaje y el rendimiento.
\end{abstract}

KEY WORDS: Incidental learning, decision making, motor control, performance, basketball.

ABSTRACT: In most of the situations arisen in basketball, the player must take decisions at a given moment within a very limited time fraction, whereas, in a simultaneous way, he carries out motor-responses also immediately. They are rapid motor-reactions taken under pressure situations, when he has no time to think twice about it (Kibele, 2006). These decisions seem to be based on intuition (Iglesias, Cárdenas \& Alarcón, 2007) and are connected to incidental learning (Poolton, Masters \& Maxwell, 2006). In sports like basketball, where time is very reduced and it is necessary to simultaneously give an immediate motor-answer, intuitive processes appear to be able to render the necessary precision and speed (Raab \& Johnson, 2008), thus improving an efficient decision-taking under stress conditions, limited time available and little information. Bearing in mind that deliberate and automatic processes work simultaneously, and that, altogether, they form up thinking an action (Betsch, 2008), we favour a model which takes into consideration both of them, since, as per Sun, Slusarz \& Terry (2005), incidental and intentional processes do coexist and they interact in order to arrive to learning and performance.

\section{Introducción}

Este estudio pretende, mediante la realización de una revisión bibliográfica, evaluar la incidencia del concepto de aprendizaje incidental en el proceso de enseñanza-aprendizaje que se produce en un deporte de interacción como el baloncesto.

En los deportes sociomotores, donde la interacción constante de todos los elementos que participan en una acción de juego configura situaciones de alta incertidumbre (Parlebás, 1988), tomar una buena decisión significa relacionar e interpretar de manera correcta todos esos elementos, seleccionando y ejecutando la forma más apropiada en el momento, lugar e intensidad adecuados, transformando de esta manera las acciones en movimientos inteligentes.

Por todo ello, la toma de decisión tendrá una enorme influencia en el rendimiento en los deportes de interacción (García, Graupera \& Ruiz, 2009; Ruiz \& Arruza, 2005), pues como afirma Antón (1998), la capacidad de pensamien-

Pablo Camacho Lazarraga, c/Rotonda Santa Eufemia 2, Edificio Horizonte, Blq 2, 4C, 41940, Tomares (Sevilla).

E-mail: pcamacholazarraga@gmail.com to táctico de los deportistas desempeña un papel decisivo en situaciones complejas de competición.

En la mayor parte de las situaciones que se producen en el baloncesto, el jugador debe tomar decisiones en un momento concreto de una fracción de tiempo muy limitada, mientras de forma simultánea ejecuta una respuesta motora también inmediata. Son reacciones motoras rápidas tomadas en situación de presión, donde el jugador no tiene tiempo para reflexionar acerca de las posibles opciones ante la situación planteada, no siendo capaz de entender los motivos por los que decide (Kibele, 2006). Estas decisiones parecen basarse en la intuición (Iglesias, Cárdenas \& Alarcón, 2007), entendiendo esto como comportamientos y conocimientos aprendidos sin intención de aprenderlos, sin que hayamos sido conscientes de haberlos aprendido cuando lo adquirimos, y sobre los que incluso actualmente podemos no tener conciencia de poseerlos (Martínez, 2004). Estas conductas se relacionan con el aprendizaje incidental (Poolton, Masters \& Maxwell, 2006), caracterizado por ser un procesamiento rápido y no verbalizable.

Por tanto, definiremos el proceso de aprendizaje inciden- 
tal como una adquisición no intencional y automática de los conocimientos sobre las relaciones estructurales entre los objetos o eventos, y aprendizaje intencional como la utilización de las instrucciones que identifican las relaciones en la tarea o los objetivos del proceso de aprendizaje, pudiendo acceder conscientemente al aprendizaje o conocimiento y comunicarlo verbalmente a otros individuos (Raab et al., 2009). Cada uno de estos procesos conducirá a mejoras en el comportamiento (Raab, 2003).

El desarrollo por tanto de ambos procesos para enseñar a los jugadores a tomar decisiones eficaces se convierte para nosotros en una tarea realmente compleja, y por tanto en un reto que debemos afrontar.

\section{Antecedentes de estudio}

Estudios como los realizados por Perales et al. (2011) y Raab (2003) se han cuestionado si el aprendizaje incidental es superior al intencional en el contexto deportivo. Uno de los temas más importantes del estudio de la intuición en la toma de decisiones es la determinación de las situaciones en que las personas puedan beneficiarse de sus respuestas intuitivas (Plessner \& Czenna, 2008). Para resolver dicha cuestión, y teniendo en cuenta que la complejidad de las situaciones servirá como factor crucial para la compresión de la ventaja de tener dos sistemas adaptativos (Raab, 2003), se han realizado diferentes estudios sobre la interacción de ambos procesos con la complejidad en la toma de decisiones de los atletas en deportes de equipo.

Cuando hablamos de aprendizaje incidental en el contexto deportivo, debemos distinguir por un lado entre aprendizaje motor incidental, es decir, de cómo producimos un movimiento específico, y por otro lado entre aprendizaje incidental cognitivo, es decir, de cómo construimos juicios acerca de la relación entre los estímulos y las acciones que se deben llevar a cabo (Masters, Law \& Maxwell, 2002). Nosotros hemos realizado este estudio en base a ambas líneas de investigación.

En relación al aprendizaje motor incidental, La forma en la que un movimiento se controla puede influir en el grado en el que el ejecutante puede combinar decisiones y movimientos con efectividad. Debido a que el comportamiento motor intencional depende de la memoria de trabajo, las demandas que resultan de los requisitos de tareas múltiples probablemente sobrecargue y perturbe su rendimiento (Masters et al., 2008). Por el contrario, será menos probable que el rendimiento motor se vea afectado si el aprendizaje se ha llevado acabo de manera incidental, pues se encontrarán disponibles más recursos para la toma de decisiones.

Trabajos como los desarrollados por Libet (1985) han demostrado que la percepción consciente del sujeto es demasiado lenta para determinar su acción. Otros trabajos, como los realizados por Masters (2000) y Poolton, Masters \& Maxwell
(2006), concluyen que si el control del movimiento se adquiere por instrucción analógica, con un bajo contenido de conocimiento declarativo, la ejecución motora no se ve afectada por las condiciones de doble tarea. Todo ello nos permite afirmar que un aprendizaje motor incidental confiere unas características de ejecución beneficiosas, tales como la robustez bajo cargas de tareas secundarias (Poolton, 2005).

En relación al aprendizaje incidental cognitivo, hablar de la toma de decisiones en el deporte supone hablar de la selección de la respuesta (Tenenbaum, 2003). Estudios realizados como los de Raab \& Johnson (2008) sobre la generación de opciones y las opciones resultantes, proporciona evidencia de la presencia, y tal vez la superioridad, de la generación de opciones intuitivas en las tomas de decisiones en los deportes de equipo. Otros estudios como los elaborados por Maxwell, Masters \& Eves (2000) y Raab (2003) concluyen de igual manera, pero ańaden que el aprendizaje incidental no es superior al intencional en relación al conocimiento de las reglas explícitas.

Gladwell (2005) afirma que cuando se toma una decisión en condiciones de mucha presión y poca información el sujeto no compara de forma lógica y sistemática todas las opciones posibles, sino que de manera inconsciente realiza una selección de datos significativos, identificando patrones a partir de fragmentos muy pequeños de experiencia.

Damasio (1994), basándose en el concepto de racionalidad limitada del premio Nobel Herbert A. Simon, que afirma que las personas generalmente no tienen ni el tiempo ni la información disponible para optimizar su capacidad cognitiva (Simon, 1955), introduce el concepto de hipótesis del marcador somático, que nos dice que las emociones tienen una función primordial en la toma de decisiones, pues marcan ciertos aspectos de una situación, o determinadas consecuencias de posibles acciones, pudiendo influir en la conclusión a favor de dicha premisa, pues como afirman Gigenenzer \& Todd (1999), las emociones pueden facilitar la toma rápida de decisiones, funcionando como principios heurísticos que guíen y detengan la búsqueda de información.

\section{Propuesta de intervención}

Tanto para unos como para otros las consignas respecto al diseño de la tarea van a ser similares. Lo que deberá cambiar es la intervención del entrenador (Cárdenas, 2009b), pues como afirman Raab \& Johnson (2008), la cantidad y la clase de instrucción, así como la organización de las situaciones de práctica, llevarán a presentaciones diferentes del conocimiento táctico y cantidad de transferibilidad entre situaciones diferentes, determinarán por tanto el estilo de aprendizaje.

En ambos casos se deberán manipular las condiciones de práctica para provocar distintos tipos de procesamientos. Tal y como afirman Iglesias et al. (2007), por un lado el entre- 
nador les dará a los jugadores información que les orienten y ayuden a interpretar mejor el juego y decidir convenientemente, provocando la reflexión o aportando información determinante sobre las claves de la decisión, favoreciendo de esta manera el procesamiento consciente (cuando hay tiempo), y por otro lado el entrenador solo diseńará y propondrá la tarea, sin proporcionar ninguna clase de feed-forward, esperando que el sujeto explore y busque las respuestas de manera libre (cuando el tiempo es mínimo).

\section{Discusión}

Debemos orientar las tareas hacia el desarrollo de los procesos cognitivos implicados en la toma de decisión en baloncesto, pues como afirman Hernández Moreno (1996) y Giménez (2000), la formación técnica en los deportes de equipo está totalmente influenciada por las conductas de decisión en la elección de la técnica adecuada para solucionar situaciones complejas del juego, pues todas las acciones estarán determinadas por la solución táctica.

Teniendo en cuenta tanto las características mencionadas anteriormente de ambos procesos de aprendizaje como los resultados obtenidos por los diferentes estudios realizados, podemos concluir que en un deporte como el baloncesto donde el tiempo es muy reducido y se necesita ejecutar de manera simultánea una respuesta motora inmediata, los procesos intuitivos parecen que pueden proporcionar la exactitud y la rapidez deseada (Raab \& Johnson, 2008), favoreciendo que se produzca una toma de decisión eficaz en condición de estrés, con límite de tiempo y poca información.

Por otro lado, estos mismos autores defienden que cuando uno habla de toma de decisiones intuitiva o deliberada no significa necesariamente que el protocolo se realice de forma completamente intuitiva o deliberada, sino que ambos procesos serán polos de una serie continua, pues como afirma Betsch (2008), es muy difícil encontrar una situación en la que se desarrolle un solo tipo de aprendizaje, con lo que los procesos automáticos y deliberados funcionarán simultáneamente, formando en su conjunto el pensamiento y la acción. aprendizaje y el rendimiento.

Nosotros abogamos por un modelo integrado de aprendizaje que tenga en cuenta tanto los procesos incidentales como los procesos intencionales, pues como afirman Sun, Slusarz \& Terry (2005), ambos tipos de procesos y conocimientos coexisten e interactúan entre sí para formar el aprendizaje y el rendimiento.

\section{Referencias}

Antón, J.L. (1998). Balonmano. Táctica grupal ofensiva. Concepto, estructura y metodología. Madrid: Gymnos.

Antón, J.L. (2000). Balonmano. Perfeccionamiento e investigación. Barcelona: Inde.

Betsch, T. (2008). The nature of intuition and its Neglect in research on judgment and decision making. En H. Plessner, C. Betsch \& T. Betsch, Intuition in judgment and decision making (pp. 3-22). New York: Lawrence Erlbaum Associates.

Cárdenas, D. (2009a). Bases para la construcción de una progresión didáctica. En G. Ortega \& A.C. Jiménez (coord.), Táctica y técnica en la iniciación al baloncesto. Sevilla:Wanceulen.

Cárdenas, D (2009b). La toma de decisiones en Baloncesto: bases neuropsicológicas y su aplicación práctica. En A. Lorenzo, S. Ibañez \& E. Ortega, Aportaciones teóricas y prácticas para el baloncesto del futuro (pp. 131-150). Sevilla: Wanceulen.

Damasio, A. (2006, 3a edición). El error de Descartes. Barcelona: Crítica.

García, V., Graupera, J.L. \& Ruíz, L.M. (2009). Perfiles decisionales de jugadores y jugadoras de voleibol de diferente nivel de pericia. Ricyde, 5 (14), 123-137.

Gigerenzer, G., \& Todd, P. (1999). Fast and frugal heuristics. En G. Gigerenzer \& P. Todd. Simple heuristics that make us Smart (pp. 3-34). New York: Oxford. U. Press.

Giménez, F.J. (2000). Fundamentos básicos de la iniciación deportiva en la escuela. Sevilla:Wanceulen.

Gladwell, M. (2005. Inteligencia intuitiva. ¿Por qué sabemos la verdad en dos segundos?. Madrid: Taurus

Hernández, J (1996). Técnica, táctica y estrategia en el deporte. Revista de Entrenamiento Deportivo, X(1), 19-22.

Iglesias, D., Cárdenas, D. \& Alarcón, F. (2007). La comunicación durante la intervención didáctica del entrenador. Consideraciones para el desarrollo del conocimiento táctico y la mejora en la toma de decisiones en baloncesto. Ciencia, Cultura y Deporte, 3 (7), 43-50.

Kibele, A. (2006). Non-consciously controlled decision making for fast motor reactions in sports. A priming approach for motor responses to non-consciously perceived movement features. Psychology of Sport and Exercise, 7, 591-610.

Libet, B. (1985). Unconscious cerebral initiative and the role of conscious will in voluntary action. The Behavioral and Brain Sciences, 8, 529-566.

Martinez, J.M. (2004). Aprendizaje implícito y explícito de secuencias: determinantes e indicadores. (Tesis doctoral no publicada). Universidad de Granada.

Masters, R. S. W., Law, J., \& Maxwell, J. (2002). Implicit and explicit learning in interceptive actions. En K. Davids, G. Savelsbergh \& J. Van Der Kamp (Eds.), Interceptive actions in sport: Information and movement (pp. 126-143). London: Routledge.

Masters, R., Poolton, J., Maxwell, J. \& Raab, M. (2008). Implicit motor learning and complex decision making in time-constrained environments. Journal of Motor Behavior, 40 (1), 71-79.

Maxwell, J.P., Masters, R.S.W. \& Eves, F.F. (2000). From novice to no know-how: a longitudinal study of implicit motor learning. Journal of Sports Sciences, 18, 111-120. Parlebás, P. (1988). Elementos de sociología del deporte. Colección Unisport. Málaga: Junta de Andalucía.

Perales, C., Cárdenas, D., Piñar, M.I., Sánchez, G. \& Courel, J. (2011). El efecto diferencial de la instrucción incidental e intencional en el aprendizaje de las condiciones para la decisión de tiro en baloncesto. Revista de Psicología del Deporte, 20(2), 729-745.

Plessner, H. \& Czenna, S. (2008). The benefits of intuition. En H. Plessner, C. Betsch \& T. Betsch. Intuition in judgment and decision making (pp. 251- 265). New York: Lawrence Erlbaum Associates.

Poolton, J., Masters, R. \& Maxwell, J. (2005). The relationship between initial errorless learning conditions and subsequent performance. Human Movement Science, 24, 362-378.

Poolton, J.M., Masters, R.S. \& Maxwell, J.P. (2006). The influence of analogy learning on decision-making in table tennis: Evidence from behavioural data. Psychology of Sport and Exercise, 7, 677-688.

Raab, M. (2003). Decision making in sports: influence of complexity on 
implicit and explicit learning. Journal of Sport and Exercise, 1, 310-337.

Raab, M. \& Johnson, J. G. (2008). Implicit Learning as a Means to Intuitive DecisionMaking inSports. En J. Plessner, C. Betsch \& T. Betsch (Eds), Intuition in Judgment and Decision Making (pp. 119-134). Nueva York: Lawrence Erlbaum Associates. Fd

Raab, M., Masters, R. S. W., Maxwell, J., Arnold, A., Schlapkohl, N. \& Poolton, J. (2009). Discovery Learning in Sports: Implicit or Explicit Processes? International Journal of Sport and Exercise Psychology, 7, 413430.

Reber, A.C. (1967). Implicit learning of artificial grammars. Journal of Verbal Learning and Verbal Behavior, 5, 855-863.
Ruiz, L.M. \& Arruza, J. (2005). El proceso de toma de decisiones en el deporte. Clave de la eficiencia y el rendimiento óptimo. Barcelona: Paidós.

Simon, H.A. (1955). A behavioral model of rational choice. The Quarterly Journal of Economics, 69(1), 99-118.

Sun, R., Slusarz, P. \& Terry, C. (2005). The interaction of the explicit and the implicit in skill learning: a dual-process approach. Psychological Review, 1 (112), 159-192.

Tenebaun, G. (2003). An integrated approach to decision making. En J.L. Strakes \& K.A. Ericsson, Expert performance in spots: advances in research on sport expertise. Champaign: Human Kinetics. 